\title{
The MeerKAT Absorption Line Survey (MALS)
}

N. Gupta*1, R. Srianand ${ }^{1}$, W. Baan ${ }^{2}$, A. Baker ${ }^{3}$, R. Beswick ${ }^{4}$, S. Bhatnagar ${ }^{5}$, D. Bhattacharya $^{1}$, A. Bosma ${ }^{6}$, C. Carilli ${ }^{5}$, M. Cluver ${ }^{7}$, F. Combes ${ }^{8}$, C. Cress $^{9}$, R. Dutta ${ }^{1}$, J. Fynbo $^{10}$, G. Heald ${ }^{11}$, M. Hilton ${ }^{12}$, T. Hussain ${ }^{1}$, M. Jarvis ${ }^{7,13}$ G. Jozsa ${ }^{14}$, P. Kamphuis $^{15}$, A. Kembhavi ${ }^{1}$, J. Kerp ${ }^{16}$, H.-R. Klöckner ${ }^{17}$, J. $\operatorname{Krogager}^{18}$, V. Kulkarni ${ }^{19}$, C. Ledoux ${ }^{20}$, A. Mahabal ${ }^{21}$, T. Mauch ${ }^{14}$, K. Moodley ${ }^{12}$, E. Momjian ${ }^{5}$, R. Morganti ${ }^{2}$, P. Noterdaeme $^{18}$, T. Oosterloo ${ }^{2}$, P. Petitjean ${ }^{18}$, A. Schröder ${ }^{22}$, P. Serra ${ }^{23}$, J. Sievers ${ }^{12}$, K. Spekkens $^{24}$, P. Väisänen ${ }^{22}$, T. van der Hulst ${ }^{25}$, M. Vivek ${ }^{26}$, J. Wang ${ }^{11}$, O.I. Wong ${ }^{27}$ and A.R. Zungu ${ }^{12}$

${ }^{1}$ Inter-University Centre for Astronomy and Astrophysics, India; (see Appendix A for author affiliations.)

E-mail: ngupta@iucaa.in

\begin{abstract}
Deep galaxy surveys have revealed that the global star formation rate (SFR) density in the universe peaks at $1 \leq z \leq 2$ and sharply declines towards $z=0$. But a clear picture of the underlying processes, in particular the evolution of cold atomic $(\sim 100 \mathrm{~K})$ and molecular gas phases, that drive such a strong evolution is yet to emerge. MALS is designed to use MeerKAT's L- and UHF-band receivers to carry out the most sensitive $\left(\mathrm{N}(\mathrm{H} \mathrm{I})>10^{19} \mathrm{~cm}^{-2}\right)$ dust-unbiased search of intervening $\mathrm{H}$ I $21-\mathrm{cm}$ and $\mathrm{OH} 18-\mathrm{cm}$ absorption lines at $0<z<2$. This will provide reliable measurements of the evolution of cold atomic and molecular gas cross-sections of galaxies, and unravel the processes driving the steep evolution in the SFR density. The large sample of H I and $\mathrm{OH}$ absorbers obtained from the survey will (i) lead to tightest constraints on the fundamental constants of physics, and (ii) be ideally suited to probe the evolution of magnetic fields in disks of galaxies via Zeeman Splitting or Rotation Measure synthesis. The survey will also provide an unbiased census of $\mathrm{H} \mathrm{I}$ and $\mathrm{OH}$ absorbers, i.e. cold gas associated with powerful AGNs $\left(>10^{24} \mathrm{~W} \mathrm{~Hz}^{-1}\right)$ at $0<z<2$, and will simultaneously deliver a blind $\mathrm{H}$ I and $\mathrm{OH}$ emission line survey, and radio continuum survey. Here, we describe the MALS survey design, observing plan and the science issues to be addressed under various science themes.
\end{abstract}

MeerKAT Science: On the Pathway to the SKA,

25-27 May, 2016,

Stellenbosch, South Africa

${ }^{*}$ Speaker. 


\section{Background}

In recent years, tremendous efforts have been dedicated to establish the evolution of the global comoving SFR density up to $z \sim 8$. The observations show that there is a peak in the comoving SFR density between $1<z<3$, followed by an order of magnitude decrease towards $z \sim 0$ over the last $10 \mathrm{Gyrs}$, i.e. $70 \%$ of the age of the Universe [1]. But a clear picture of the underlying processes that drive such a strong evolution is yet to emerge.

Since stars form in molecular clouds, the observations of the molecular gas $\left(\mathrm{H}_{2}\right)$, which is the basic fuel for star formation, and the atomic gas ( $\mathrm{H} \mathrm{I}$ ), which is the dominant gas component of galactic discs, can be used to understand the evolution of SFR density. The atomic gas component of the interstellar medium (ISM) is easily observable through the $\mathrm{H}$ I $21-\mathrm{cm}$ emission line. But due to technical limitations the $\mathrm{H}$ I $21-\mathrm{cm}$ emission line studies at present are mostly confined to the local Universe. Through the observations of $\mathrm{H} \mathrm{I} 21-\mathrm{cm}$ emission line in the local Universe and the large samples of damped Ly $\alpha$ absorbers (DLAs ${ }^{1}$ ) at high- $z$, the H I mass density $\left(\Omega_{\mathrm{HI}}\right)$ of the Universe is reasonably well-constrained at $z<0.2$ and $2<z<4$, respectively. They reveal a very modest (factor $\sim 2$ ) decrease in $\Omega_{\mathrm{HI}}$ compared to an order of magnitude decrease in the SFR density over the same redshift range. This implies that the processes leading to the conversion of atomic gas to molecular gas and eventually to stars need to be understood directly via observations of cold atomic and molecular gas, rather than the evolution of $\Omega_{\mathrm{HI}}$.

It is well known that $\mathrm{H} \mathrm{I} 21-\mathrm{cm}$ absorption is an excellent tracer of the cold neutral medium (CNM; T $100 \mathrm{~K}$ ) in the Galaxy. Similarly, the OH radical is one of the common constituents of diffuse and dense molecular clouds, and can be observed through the two main lines at $1665 \mathrm{MHz}$ and $1667 \mathrm{MHz}$, and the two satellite lines at $1612 \mathrm{MHz}$ and $1720 \mathrm{MHz}$. As the volume filling factor of ISM phases and the observables associated with the $\mathrm{H}$ I and $\mathrm{OH}$ absorption lines depend sensitively on in situ star formation through various stellar feedback processes, these lines can be used to probe the physical conditions in the ISM and understand the cosmic evolution of the SFR density $[2,3,4,5]$. In addition, the observed frequencies of these lines can be used to place the most stringent constraints on the variation of fundamental constants of physics [6,7] and cosmic acceleration $[8,9]$.

Despite the scientific potential of intervening H I $21-\mathrm{cm}$ and $\mathrm{OH} 18-\mathrm{cm}$ lines, and the tremendous efforts from the community over last three decades, mainly due to technical limitations, only $\sim 50$ intervening $\mathrm{H}$ I $21-\mathrm{cm}[10,11]$ and 3 intervening molecular absorbers [12] at radio wavelengths are known. The small number of detections has hampered attempts to systematically map the cold gas evolution with any statistical significance [Fig. 13 of 10].

The availability of various SKA pathfinders in RFI-free environments offers an unique opportunity to overcome these limitations.

In 2010, MALS was identified as one of the ten large surveys to be carried out with MeerKAT. The main objective of the survey is to use MeerKAT's L- and UHF-band receivers to carry out the most sensitive $\left(\mathrm{N}(\mathrm{H} \mathrm{I})>10^{19} \mathrm{~cm}^{-2}\right)$ dust-unbiased search of intervening $\mathrm{H} \mathrm{I} 21-\mathrm{cm}$ and $\mathrm{OH} 18-\mathrm{cm}$ absorption lines at $0<z<2$. The survey is expected to detect $\sim 200$ intervening $21-\mathrm{cm}$ absorbers. This will provide reliable measurements of the evolution of cold atomic and molecular gas crosssections of galaxies, and unravel the processes driving the steep evolution in the SFR density. Due

\footnotetext{
${ }^{1} \mathrm{H}$ I column density, $\mathrm{N}(\mathrm{H} \mathrm{I}) \geq 2 \times 10^{20} \mathrm{~cm}^{-2}$.
} 
to the large field-of-view and excellent sensitivity of MeerKAT, the survey is also expected to detect $\sim 50021-\mathrm{cm}$ absorbers associated with AGNs. These associated and intervening absorbers detected from the survey will enable discovery through a wide range of science themes that are described in Section 2. The survey design is presented in Section 3. A high-level plan for data products, observing plan and data releases is provided in Section 4. The relationship with other SKA pathfinder surveys and key science is presented in Section 5.

\section{MALS - Key Science Themes}

The key science themes based on the survey design (see Section 3; Table 2) are as follows:

1. Evolution of cold gas in galaxies and relationship with SFR density: MALS will be sensitive to detect $\mathrm{H}$ I $21-\mathrm{cm}$ absorption and constrain the number per unit redshift range of intervening $\mathrm{H}$ I $21-\mathrm{cm}$ absorbers, $n_{21}$, from sub-DLAs to DLAs, i.e. the CNM fraction in a wide range of column densities tracing the inner disks of galaxies, as well as the extended disks, halos, intra-group gas and the circumgalactic medium (CGM). It will measure the evolution of cold atomic and molecular gas cross-section of galaxies in a luminosity- and dust-unbiased way and constrain the ISM physics at pc scales over the entire redshift range $0<z<2$. Through MALS, the detection of $\sim 200$ intervening 21-cm absorbers is expected. With these it will constrain the evolution of $n_{21}(z)$, split in 3 redshift bins over $0<z<1.5$, with $\sim 20 \%$ accuracy. If $n_{21}(z)$ scales with $\Omega_{\mathrm{HI}}(z)$, the $20 \%$ accuracy will allow us to detect the evolution in $n_{21}(z)$, and hence the CNM cross-section of galaxies, at the $3 \sigma$ level (for example, see [13]). Through $\mathrm{OH}$ lines, the survey will be sensitive to the molecular gas crosssection of galaxies, and will provide the first estimate of the number per unit redshift range of $\mathrm{OH}$ absorbers, $n_{\mathrm{OH}}(z)$. The $n_{21}(z)$ and $n_{\mathrm{OH}}(z)$ from the survey will be key observables to understand the process of condensation of warm gas into cold gas and eventually stars.

In Table 1, we compare MALS with other upcoming $\mathrm{H}$ I imaging surveys with footprint and survey depth large enough to deliver a substantial number of $21-\mathrm{cm}$ absorbers. The Westerbork Synthesis Radio Telescope (WSRT) shallow survey will search for H I emission line at $\delta>+30^{\circ}$ with Apertif, a phased-array feed for WSRT. Simultaneously, it will deliver a 21-cm absorption line survey called SHARP (PI: Morganti). The Australian SKA Pathfinder (ASKAP) will have a dedicated 21-cm absorption line survey at $0.5<z<1$ called the First Large Absorption Survey in H I (FLASH; PI: Sadler). WALLABY (PIs: Koribalski and Staveley-Smith) is the ASKAP H I emission all-sky survey at $\delta<30^{\circ}$ that will simultaneously deliver a $21-\mathrm{cm}$ absorption line survey at $z<0.26$.

From Table 1 it is clear that amongst various upcoming major $\mathrm{H}$ I $21-\mathrm{cm}$ absorption line surveys, MALS is well distinguishable. It is the only survey that will uniformly cover $0<$ $z<1.5$, the most important redshift range as far as the evolution of SFR and AGN activity are concerned (cf. columns 2-3). It also provides excellent spectral line sensitivity in relatively much less observing time per pointing (cf. columns 4-5). The last column provides the number of sources or sight lines within the survey area towards which H I 21-cm absorption from a $100 \mathrm{~K}$ gas with the DLA column density would be detectable. The flux density cutoff corresponding to this optical depth sensitivity is also provided. Note that the last column 
Table 1: Summary of various upcoming H I 21-cm absorption line surveys

\begin{tabular}{|c|c|c|c|c|c|c|c|}
\hline Survey & $\begin{array}{c}\text { Frequency } \\
\text { coverage } \\
(\mathrm{MHz})\end{array}$ & $\begin{array}{l}\text { Redshift } \\
\text { range } \\
\text { (H I 21-cm) }\end{array}$ & $\begin{array}{c}\text { Time } \\
\text { per } \\
\text { pointing } \\
\text { (hrs) }\end{array}$ & $\begin{array}{c}\text { Spectral } \\
\text { rms per } \\
\sim 5 \mathrm{~km} \mathrm{~s}^{-1} \\
(\mathrm{mJy} / \mathrm{b})^{\dagger}\end{array}$ & $\begin{array}{c}\text { Sky } \\
\text { coverage } \\
\left(\mathrm{deg}^{2}\right)\end{array}$ & $\begin{array}{l}\text { Total } \\
\text { time } \\
\text { (hrs) }\end{array}$ & $\begin{array}{c}\text { No. of } \\
\text { sight lines }\end{array}$ \\
\hline $\begin{array}{r}\text { Apertif } \\
\text { SHARP }\end{array}$ & $1130-1430$ & $0-0.26$ & 12 & 1.3 & 4000 & 6000 & $\begin{array}{c}25000 \\
(>30 \mathrm{mJy})\end{array}$ \\
\hline $\begin{array}{l}\text { ASKAP } \\
\text { FLASH }\end{array}$ & $700-1000$ & $0.5-1.02$ & 2 & 3.8 & 25000 & 1600 & $\begin{array}{c}65000 \\
(>90 \mathrm{mJy})\end{array}$ \\
\hline $\begin{array}{c}\text { ASKAP } \\
\text { WALLABY }\end{array}$ & $1130-1430$ & $0-0.26$ & 8 & 1.6 & 30000 & 8000 & $\begin{array}{c}132000 \\
(>40 \mathrm{mJy})\end{array}$ \\
\hline $\begin{array}{l}\text { MALS } \\
\text { L-band }\end{array}$ & $900-1670$ & $0-0.57$ & 1 & 0.5 & 1000 & 691 & $\begin{array}{c}12000 \\
(>15 \mathrm{mJy})\end{array}$ \\
\hline $\begin{array}{c}\text { MALS } \\
\text { UHF-band }\end{array}$ & $580-1015$ & $0.4-1.44$ & 2 & 0.6 & 700 & 746 & $\begin{array}{c}12000 \\
(>15 \mathrm{mJy})\end{array}$ \\
\hline
\end{tabular}

$\dagger$ Estimated at the center of the band; ${ }^{\star}$ See text for details.

does not take into account the redshift distribution of radio sources and any variation in gas properties with redshift or radio source type, but provides a useful metric to compare the overall effectiveness of these surveys with different characteristics. Fig. 1 shows the distribution of redshift path for intervening absorption for the same optical depth sensitivity. Here, the analysis takes into account the redshift and spatial distribution of radio sources over the full survey area. For MALS, we have considered the survey area corresponding to $20 \%$ sensitivity of the maximum of the primary beam. In summary, it is evident that except for WALLABY for which a large amount of telescope time in a narrow redshift range has been allocated to an $\mathrm{H} \mathrm{I} 21-\mathrm{cm}$ emission line survey, the MALS is comparable to other surveys at $z>0.26$. In particular, both in the L- and UHF- bands it covers more than $50 \%$ of the redshift ranges inaccessible to any of the other surveys. Notably, the redshift ranges of $0.3<z<0.5$ and $1<z<1.5$ are only accessible to MALS.

The absorbers from MALS will be followed-up with (1) VLBI to map the pc-scale structure in the ISM [14], (2) HST, SALT and VLT to detect various atomic/metal absorption lines to constrain the ambient radiation field, metallicity, density and temperature via detections, and VLA/MeerKAT to probe the magnetic fields in galactic discs via rotation measure synthesis [15]. These parameters, in addition to $n_{21}$ and $n_{\mathrm{OH}}$, will be valuable inputs to test and refine the physical models of the ISM so far based primarily on the galactic ISM [4] and understand the processes driving the evolution of SFR density. Further, the multi-wavelength follow-ups of the absorbers using ALMA, NOEMA, VLA, VLT and SALT will lead to the discovery of rare atomic and molecular species which will be used to measure the evolution of CMB temperature and constrain the effective equation of state of decaying dark energy [16].

2. Fuelling of AGN, AGN feedback and dust-obscured AGNs: AGN activity is known to peak during the same epoch as SFR density. Both theoretical and observational considera- 

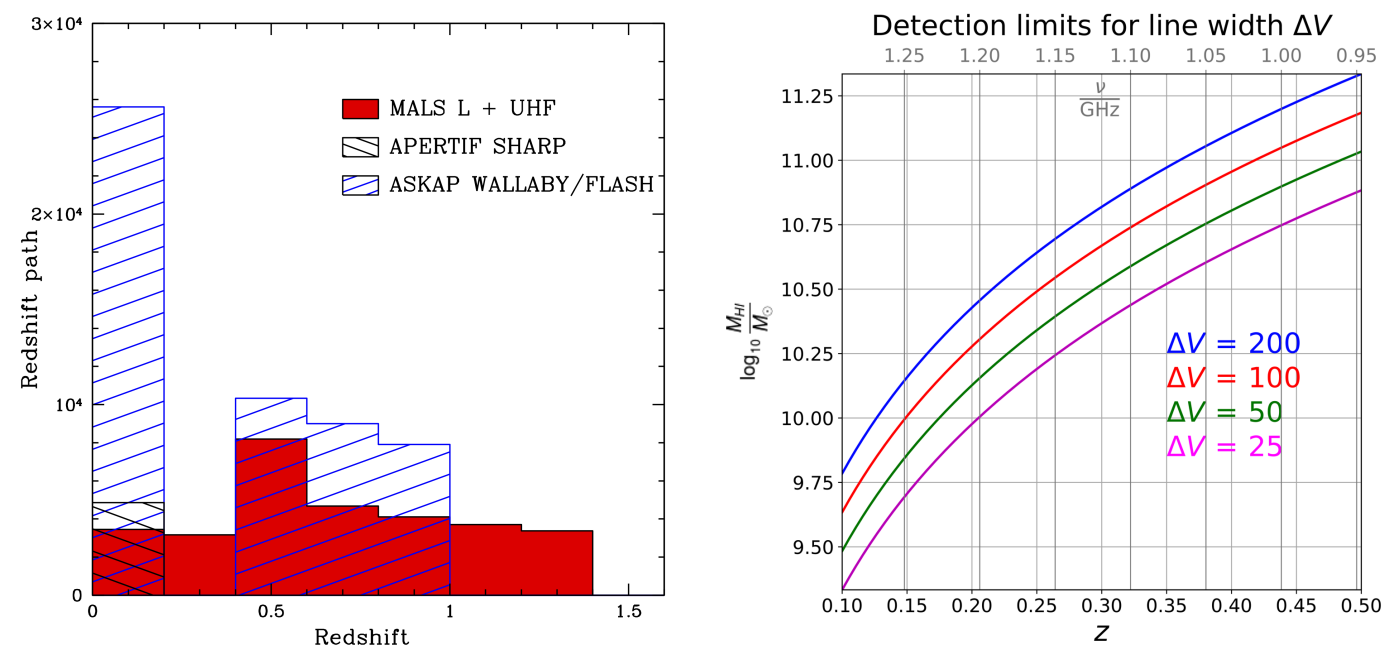

Figure 1: Left: Redshift path comparison for various absorption line surveys (also see Table 1). The MALS L- and UHF-band $\Delta z$ are estimated for the primary beams at $1200 \mathrm{MHz}$ and $800 \mathrm{MHz}$ respectively. For MALS, the steep rise in the bin at $z=0.5$ is (primarily) due to this and the fact that radio sources are brighter at lower frequencies (spectral index 0.8). Also, at $z=0.5$ bin both the L- and UHF-bands contribute as they overlap at 900-1015 MHz. Right: $5 \sigma \mathrm{H}$ I mass emission-line detection limits for the observed fields ( $\mathrm{r}$ $=0.36^{\circ}$ ) for a total observing area of $300 \mathrm{deg}^{2}$.

tions suggest a close relationship between galaxy evolution and AGN activity [17]. Further, the existence of red i.e. dust-obscured AGNs is predicted in both the orientation-based AGN unification models and the evolutionary scenarios but the exact fraction of such AGNs is still uncertain with the estimates ranging over 10-50\% [18]. Through a dust-unbiased search of $\mathrm{H} \mathrm{I}$ and $\mathrm{OH}$ absorptions, MALS will determine the (1) distribution and kinematics of atomic and molecular phases associated with circumnuclear gas and the ISM of AGN hosts, and (2) the fraction of dust obscured AGNs missed out in optical/ultraviolet surveys. Through these the survey will address several fundamental issues in the context of AGN evolution, AGN feedback and the AGN Unification paradigm.

Presently, about 100 associated $\mathrm{H}$ I 21-cm absorbers and only a few $\mathrm{OH}$ absorbers are known $[19,20,21]$. In MALS survey area, there will be $\sim 2000$ sources brighter than $30 \mathrm{mJy}(\sim 1$ $\mathrm{GHz}$ ) and suitable for the associated $21-\mathrm{cm}$ and $\mathrm{OH}$ absorption line search. Adopting a detection rate of $25 \%[21,22]$, the detection of $\sim 500$ associated $21-\mathrm{cm}$ absorbers is expected. This will provide a large sample to study the properties of cold gas in powerful AGNs $\left(>10^{24}\right.$ $\mathrm{W} \mathrm{Hz}^{-1}$ ) by splitting these into subsamples of different AGN types and redshifts. For the brighter sources, the survey will also be sensitive to detect rare blue-shifted $\mathrm{H}$ I wings in the absorption profile [23]. Such wings representing outflows have been identified in $\sim 5 \%$ of the AGNs observed at $z<0.2$. The evolution of this fraction and the mass-outflow rate with redshift is a key observable to compare with the predictions from the models of galaxy evolution. MALS will extend the observations of these outflows to a largely unexplored $(z>0.2)$ and the most relevant redshift range in this context.

3. Galaxy evolution through $\mathbf{H}$ I and $\mathbf{O H}$ emission: With an integration time of $56 \mathrm{~min}-$ 
utes per pointing in L-band, MALS is a substantial blind emission line survey. MALS provides a set of 740 quasi-random pointings (with a potential sub-sample pointing towards regions of high interest such as galaxy groups). With the natural rms of $0.26 \mathrm{mJy}^{\text {beam }}{ }^{-1}$ over $20 \mathrm{~km} \mathrm{~s}^{-1}$ at the pointing centre delivered by MALS, a galaxy like Leo T with a dark matter mass that is just enough to retain baryons, $\mathrm{M}(\mathrm{H} \mathrm{I}) \sim 2.8 \times 10^{5} \mathrm{M}_{\odot}$ and radius $\sim 240 \mathrm{pc}$ [24], would be detectable above a $5 \sigma$ level and resolved to a distance of $4 \mathrm{Mpc}$. Over an area of $300 \mathrm{deg}^{2}$, MALS will be able to detect an $M_{*}$ galaxy up to a redshift of $z \sim 0.1-0.2$ and the galaxies with the largest $\mathrm{H}$ I masses out to $z>0.5$ (see Fig. 1). We estimate the number of detections to be of the order of 5000-15000 galaxies, many of which will be resolved. In addition, tenuous gas at column densities of $0.2 \mathrm{M}_{\odot} \mathrm{pc}^{-2}$ will be detectable over $20 \mathrm{~km} \mathrm{~s}^{-1}$ at the $6 \sigma$ level at $45^{\prime \prime}$ resolution. MALS will therefore probe the gas distributions and kinematics of a substantial number of galaxy disks while providing an unique opportunity to relate these to the cold atomic and molecular gas detected through $\mathrm{H}$ I and $\mathrm{OH}$ absorption.

The main goals of MALS as an H I emission line survey are: (1) to catalog basic emission line parameters of observed objects, (2) to produce $\mathrm{H}$ I emission line maps and data cubes of resolved emission line objects, (3) to parameterize the extended morphology and kinematics, spin characteristics, rotation curves, and surface density profiles of the resolved galaxies independently as well as in connection with the absorption line survey to understand the nature of $21-\mathrm{cm}$ and $\mathrm{OH}$ absorbers, and (4) to study velocity and mass functions, (dark) matter distribution and quantify accretion properties. With these observables MALS will be a perfect emission line progenitor survey for already addressing major questions raised in the SKA science book: (1) studying the radial gas profiles of galaxies in the light of galaxy evolution [26], (2) studying the matter distribution in galaxies, (3) quantifying the intrinsic and mutual spin distribution in galaxies and its dependence on their environment [27], and (4) studying the low column density environment of galaxies to quantify (AGN) feedback and accretion processes on a statistical basis [28].

Besides H I emission, MALS will also be capable of detecting OH Megamasers (MMs). Powerful OH MMs have already been detected up to a redshift of 0.265 (1318 MHz; [25]) and the emission profiles show strong outflows and evidences of strong interactions. The population of sources will increase at least up to a redshift of 2.5 because of the link between ULIRG $^{2}$ host galaxies and the increasing merger rate with redshift. OH MMs are a good tracer of starburst activity and merger history and MALS will establish their luminosity function at higher luminosities and redshifts.

4. Radio continuum and polarization science with MALS: Magnetic fields are known to be present over a wide range of physical scales in astrophysical objects. They are an important component in the energy balance of the interstellar medium (ISM) of galaxies. Besides being important in determining the conditions for the onset of star formation in protostellar clouds they are also relevant for (1) the formation and stabilisation of gas disk and spiral arms, (2) the heating of gas, especially, in the outer disks and halos, and (3) the launching of galactic outflows and determining how the matter and energy are distributed throughout the galaxy.

\footnotetext{
${ }^{2}$ Ultraluminous infrared galaxy
} 
Overall, magnetic fields are tightly coupled to various stellar feedback processes and play an important role in a wide range of physical processes that drive galaxy evolution. MALS will provide extremely sensitive wide-band L- and UHF-band observations to observe total intensity and polarized emission from a large number of normal and active galaxies enabling wide range of projects some of which are described below.

Clusters, halos and relics: MALS will allow us to conduct a blind survey of a statistically significant number of galaxy clusters to discover new diffuse cluster emission and constrain their formation models through spectral studies. We expect to detect about 770 clusters with $\mathrm{M}_{500}>3 \times 10^{14} \mathrm{M}_{\odot}$ within the survey region, where $\mathrm{M}_{500}$ is the cluster mass within a density five hundred times the critical density. All MALS pointings will be within the footprint of the Advanced ACT cosmic microwave background survey [29], in which MALS team members are actively involved, and which will deliver Sunyaev-Zel'dovich masses for all clusters above this mass limit. With the $\sim 3 \mu \mathrm{Jy}$ flux density limit of MALS, we expect to observe diffuse radio halo emission in approximately 80 of these clusters, over a wide range of cluster mass and redshift $(0<z<0.8)$. This will provide a large enough statistical sample to study the evolution of radio halo properties, which significantly extends the existing heterogeneous sample of about 50 giant radio halos [30] beyond the current range of masses $\left(\mathrm{M}_{500}>5 \times 10^{14} \mathrm{M}_{\odot}\right)$ and redshifts $(0.2<z<0.4)$. We will place constraints on radio halo formation models, by measuring fluxes and spectral indices over the wide MeerKAT frequency bands, as well as distinguish between turbulent halos generated by re-acceleration during cluster mergers, and off-state halos of a purely hadronic origin found in more relaxed systems [31]. The polarization data from MALS will be crucial for a full study of the relic emission by testing the shock- related formation theories through inferred Mach numbers and magnetic field orientations.

Magnetic fields in galaxies and AGNs: With the sensitivity of $\sim 3 \mu \mathrm{Jy}$, we expect more than 30 polarized sources per square degree at $1.4 \mathrm{GHz}$ [32]. The wide-band coverage of MALS will allow us to quantify the effects of depolarization and measure currently unknown polarized source counts at low frequencies. The strong intervening $21-\mathrm{cm}$ absorbers detected through MALS will be an excellent tracer of galactic discs and ideally suited to probe the evolution of magnetic fields in galaxies by rotation measure synthesis. Until now, such studies using samples of Mg II absorbers have yielded ambiguous results [15] because of the small sample sizes and the difficulty in interpreting the origin of $\mathrm{Mg}$ II absorbers. Additionally, the sizes and structures of radio sources are often found to be affected by the ambient gas in the central regions of host galaxies, suggesting strong dynamical interaction with the external medium. Evidence of this gas may also be probed via polarization measurements and will be used to understand the role of jet-mode feedback in driving the outflows detected in 21-cm absorption and the evolution of AGNs.

5. Constraining space- and time-variation of fundamental constants: The absorption lines seen in the spectra of distant QSOs can be used to place constraints on the space and time variations of different dimensionless fundamental constants of physics [33]. For the past decade or so based on systematic efforts using high resolution echelle spectrographs at VLT and Keck, the community has constrained the variation of $\alpha=\left(e^{2} / h c\right)$ and $\mu=\left(m_{e} / m_{p}\right)$ at 
Table 2: MALS Survey Design

\begin{tabular}{cccccc}
\hline MALS phase & $\begin{array}{c}\text { Number } \\
\text { of pointings }\end{array}$ & $\begin{array}{c}\text { Time } \\
\text { per pointing } \\
(\text { mins })\end{array}$ & $\begin{array}{c}\text { Spectral } \\
\mathrm{rms}^{\dagger} \\
\left(\mathrm{mJy} \mathrm{beam}^{-1}\right)\end{array}$ & $\begin{array}{c}\text { Continuum } \\
\mathrm{rms} \\
\left(\mu \mathrm{Jy} \mathrm{beam}^{-1}\right)\end{array}$ & $\begin{array}{c}\text { Total on-source } \\
\text { time } \\
(\mathrm{hrs})\end{array}$ \\
\hline $\begin{array}{c}\text { L-band } \\
(900-1670 \mathrm{MHz}) \\
\text { UHF-band }\end{array}$ & 340 & 56 & 0.5 & 3 & 691 \\
$(580-1015 \mathrm{MHz})$ & 370 & 121 & 0.6 & 3 & 746 \\
\hline
\end{tabular}

${ }^{\dagger}$ Estimated at $\sim 1200 \mathrm{MHz}$ and $\sim 800 \mathrm{MHz}$ for the full band split into 32768 channels.

the level of $1 \mathrm{ppm}$ at high-redshift. The next major step is to improve these constraints by another factor of 10 i.e. 1 part in $10^{7}$ which will be comparable to local measurements based on atomic clocks. The major systematics that prevents any further improvement stems from the stability of wavelength scales in present day optical spectrographs [34]. Further progress is possible through radio absorption lines because the frequency scales at radio telescopes are known to be well-defined and compared to optical/ultraviolet lines the radio lines are more sensitive to the variation of fundamental constants. But a large sample of radio absorbers is required to achieve this so that any systematics introduced due to ionisation, radiation and excitation inhomogeneities in the gas that gets reflected as the variation of constants is randomised. MALS will provide such a sample for the first time. Through main- and satellite- $\mathrm{OH}$ absorption lines from the survey, or the joint analysis of $21-\mathrm{cm}$ and $\mathrm{OH}$ absorption with various metal, atomic and molecular absorption lines detected through follow-ups with ALMA, NOEMA, VLA, VLT and SALT the survey will lead to tightest constraints on the variations of fundamental constants of physics.

6. Stacking line, continuum and polarization data: Stacking of undetected H I $21-\mathrm{cm}$ line spectra of sources with known redshifts has become a tested method to study the atomic gas in galaxies [35]. We will apply stacking technique to spectral line, radio continuum and polarization data from MALS to make measurements that would otherwise be possible only with much deeper observations or SKA-I. In H I and OH absorption, MALS will likely detect galaxies and AGNs undetected at other wavelengths. This will not only constrain the redshift of such sources but also investigate their multi-wavelength properties by stacking.

\section{Survey Design}

The MALS survey design is summarized in Table 2. The total L- and UHF-band on-source times for 740 and 370 pointings are 691 and $746 \mathrm{hrs}$ respectively. Assuming $15 \%$ overheads this corresponds to a total observing time of $1655 \mathrm{hrs}$. The survey strategy that yields almost uniform redshift path coverage over the entire redshift range of interest (see Fig. 1) is based on the optimization of the following two parameters that drive the number of detections and the science output from the survey: 
1. Optical depth sensitivity $\left(\int \tau \mathrm{dv}\right)$ : The target $5 \sigma$ integrated optical depth sensitivity for intervening H I $21-\mathrm{cm}$ absorption at the center of the primary beam is $\int \tau \mathrm{dv}=0.045 \mathrm{~km} \mathrm{~s}^{-1}$. This corresponds to a sensitivity to detect the CNM (100 K) in N(H I $\sim 10^{19} \mathrm{~cm}^{-2}$ gas via H I $21-\mathrm{cm}$ absorption, and is essential to detect the CNM in a wide range of physical environments. This will be achieved by centering L- and UHF-band pointings on bright ( $>400 \mathrm{mJy}$ at $1 \mathrm{GHz}$ ) radio sources.

2. Redshift coverage/ Survey redshift path $(\Delta z)$ : As the sensitivity to detect the CNM down to a $\mathrm{H} \mathrm{I}$ column density of $10^{19} \mathrm{~cm}^{-2}$ will be achieved only towards the central source, it is essential that these be selected carefully. The MeerKAT L- and UHF-band receivers cover frequency ranges of $900-1670 \mathrm{MHz}$ and $580-1015 \mathrm{MHz}$ respectively. For the $\mathrm{H} \mathrm{I} 21-\mathrm{cm}$ line, these correspond to a redshift coverage of $0<z<0.58$ and $0.40<z<1.44$, respectively ${ }^{3}$. The redshift path for central bright sources will be maximized by selecting these to be at $z>0.6$ and $z>1.4$, respectively. With this approach, based on the $n_{21}$ from [10], which has $\sim 50 \%$ errors, we expect to detect $\sim 100$ intervening $21-\mathrm{cm}$ absorbers towards the central bright sources (we adopt $n_{21}=0.13 ; \Delta \mathrm{z}=800$ and assume no redshift evolution).

The spectral $\mathrm{rms}$ of $\sim 0.5 \mathrm{mJy}$ would also ensure that an adequate redshift path to deliver a similar number of intervening $21-\mathrm{cm}$ absorbers with $\mathrm{N}(\mathrm{H} \mathrm{I}) \sim 10^{20} \mathrm{~cm}^{-2}$ (i.e. $\int \tau \mathrm{dv}=0.5 \mathrm{~km} \mathrm{~s}^{-1}$ for $100 \mathrm{~K}$ ) is achieved towards off-axis weaker sources, and the sight lines with rare high column density absorbers are not under-represented in the survey.

Over the last few years results from several H I $21-\mathrm{cm}$ absorption line searches in the samples of Mg II absorbers at $0.5<z<1.5[10,36]$, DLAs at $z>2[11,37,38]$ and quasar-galaxy pairs (QGPs) at $z<0.3[39,40,41,42]$ have become available. From VLBA imaging and spectroscopy of a subset of these samples it has become increasingly obvious that higher detection rates of intervening 21-cm absorption are achieved for flat-spectrum radio sources (see Fig. 6 of [10]). This is related to the typical correlation length of 30-100 pc exhibited by the CNM gas (see also [43]). The gas clouds are expected to be much more compact $(<10 \mathrm{pc})$ in the case of translucent or dense phases of the ISM [14]. Therefore, for a blind search of intervening absorbers the background sources have to be as compact as possible. The flat-spectrum radio quasars (FSRQs), which are known to be compact, are hence the straightforward choice for MALS.

Thus, in addition to the criteria that the central sources for L- and UHF-band pointings be at $z>0.6$ and $z>1.4$, respectively, we further require that a majority of these be FSRQs.

\section{Survey Strategy, Observing Plan and Data Releases}

The survey is expected to start in 2018 and will be carried out over a period of five years. Based on the outcomes from the first year of observations, the proportion of L- and UHF-band pointings for the survey will be revised.

\section{Survey strategy and observing setup}

1. Observing setup: The data will be acquired in both parallel- and cross-polarization hands using a correlator dump time of $4 \mathrm{~s}$. The $\mathrm{L}(900-1670 \mathrm{MHz})$ and UHF $(580-1015 \mathrm{MHz})$

\footnotetext{
${ }^{3}$ The corresponding redshift ranges for $\mathrm{OH}$ main lines are $0<z<0.85$ and $0.64<z<1.87$, respectively.
} 
bands will be split into 32768 frequency channels. This would deliver a spectral resolution of $\sim 5 \mathrm{~km} \mathrm{~s}^{-1}$, and will be adequate to detect and resolve the structure in intervening $21-\mathrm{cm}$ absorption lines (e.g. see Fig. 12 of [44]).

2. Calibration overheads: For a two-hour observing block we assume a calibration overhead of $15 \%$. This includes observations of a standard calibrator every two hours and a 2 min scan on a phase calibrator every 45 mins for flux density scale and bandpass calibrations. The leakage calibration will be carried out through observations of an unpolarized calibrator. We will also require a polarization angle calibrator. It is quite possible that the polarization angle can be calibrated using data from previous days, and that there will be no need to observe a separate phase calibrator. All this will be tested and confirmed during the commissioning phase.

3. Observing blocks and Scheduling: The time spent on each pointing ranges from 56 to 121 mins. The total time on each pointing may be split into two equal sessions, which will be executed within a few months with a gap that ensures a shift of $20-30 \mathrm{~km} \mathrm{~s}^{-1}$ in the absorption lines. This shift due to the heliocentric motion of the Earth would allow us to unambiguously distinguish between true absorptions and RFI, and is required to build a reliable absorption line catalog. We intend to execute these sessions through observing blocks of one to several hours.

4. Cadence: Each pointing will have two epochs separated by a few months. The pointings ( $\sim 300)$ that are common between the L- and UHF-band phases will have four epoch observations.

5. Strategy for the selection of pointings: For the first year of observations, we will select $70 \%$ of the pointings at $-40^{\circ}<\delta<+30^{\circ}$ and $100 \%$ of these will be centered at FSRQs with spectroscopically confirmed redshifts. This will allow accurate determination of redshift path length functions towards the central bright source and lead to the first reliable constraints on $n_{21}(z)$ and $n_{O H}(z)$. The availability of VLBI images for all of the central targets in the first year of observations will allow us to quantify the effect of radio structure on the detectability of absorption and its redshift evolution. All this will allow us to use first year's observations as a proving ground and, if needed, alter the choice of pointings for the subsequent phases.

Bright FSRQs with known spectroscopic redshifts, especially in the southern hemisphere, are rare. Therefore, we are carrying out an ambitious optical spectroscopic campaign using SALT and NOT to increase the number of FSRQs with $z>1.4$. For this, using WISE IR colors we have developed a pre-selection method that allows us to select $z>1.4$ 'candidate' quasars with $\sim 75 \%$ efficiency (Fig. 2). Through this ongoing program we have already identified $\sim 100$ new FSRQs over last two years.

All the data products from the survey (i.e. calibrated visibilities and images) will be released to community through regular data releases. 


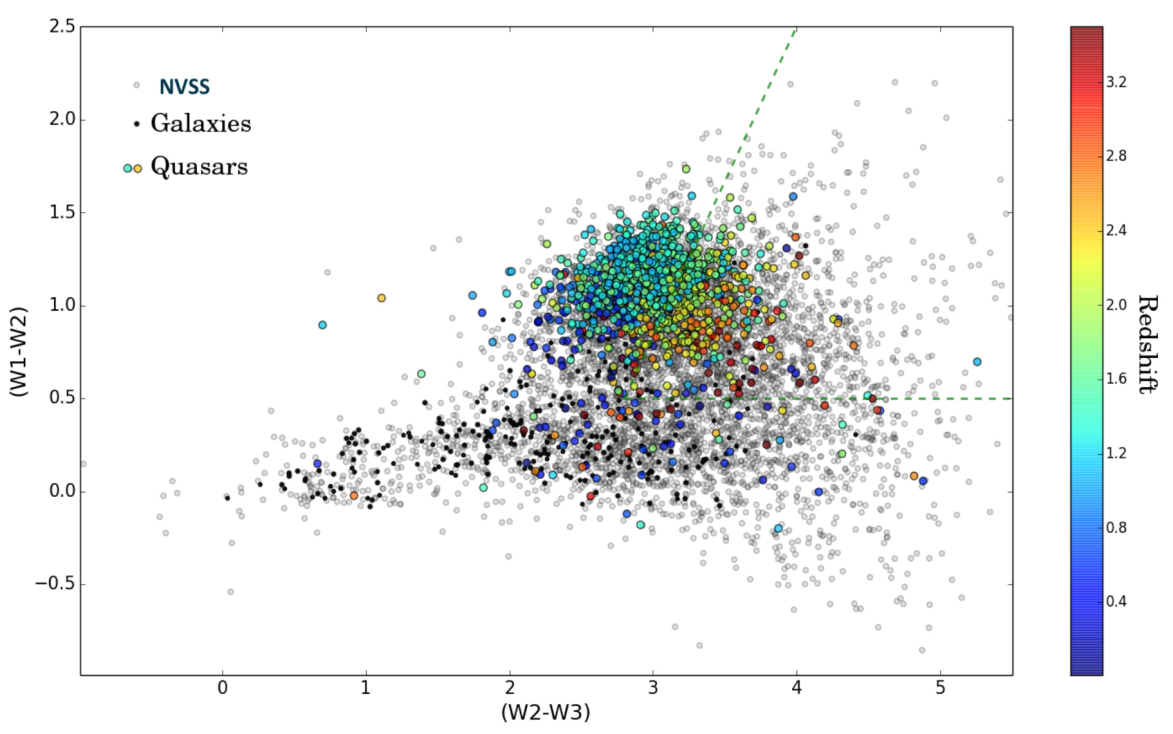

Figure 2: WISE IR colors for radio sources $\left(\delta<+20^{\circ},>200 \mathrm{mJy}\right)$ (grey). Galaxies identified in the literature are shown as small black points and the known quasars are color coded as a function of redshift. The dashed lines indicate the color-color space for selection of $z>1.4$ radio loud quasars.

\section{Relationship with other SKA Pathfinder surveys}

Understanding galaxy evolution through H I $21-\mathrm{cm}$ line is one of the key science drivers of the SKA [45, 46]. MALS will measure the evolution of the cold atomic and molecular gas crosssections of galaxies in a luminosity- and dust-unbiased way and constrain the ISM physics at pc scales over the entire redshift range: $0<z<2$ (cf. Table 1 for complementarity with other 21$\mathrm{cm}$ absorption line surveys). Through the excellent sensitivity of MeerKAT over a wide range of spatial resolutions and wideband spectro-polarimetry, MALS will simultaneously deliver a highly competitive blind $\mathrm{H}$ I/ $\mathrm{OH}$ emission line and radio continuum survey, and will address a wide range of science issues raised in the SKA science book. The survey will also complement H I $21-\mathrm{cm}$ emission line surveys such as (1) LADUMA and WALLABY that will be affected by luminosity bias and will probe only most luminous objects at higher redshifts, and (2) MHONGOOSE that will measure the sub-kpc scale properties of gas but in a sample of nearby galaxies. The conversion of gas into stars involves complex physical processes from pc to kpc and even Mpc scales. Together, these surveys will provide observational constraints on the properties of cold gas and the underlying physical processes at all physical scales required to understand the evolution of SFR density and design the future H I 21-cm line surveys with SKA.

\section{Acknowledgments}

We thank Kenda Knowles of UKZN for useful discussions. We thank IUCAA, Rutgers University and the South African members for the SALT observing time contributed to the survey to identify high- $z$ quasars for MALS. NOT is operated by the Nordic Optical Telescope Scientific Association at the Observatorio del Roque de los Muchachos, La Palma, Spain, of the Instituto de Astrofisica 
de Canarias. We acknowledge the support from ThoughtWorks India Pvt. Limited in developing ARTIP - a prototype of MALS data analysis pipeline.

\section{References}

[1] R. J. Bouwens, L. Bradley, A. Zitrin, D. Coe, M. Franx, W. Zheng et al., A Census of Star-forming Galaxies in the Z 9-10 Universe based on HST+Spitzer Observations over 19 Clash Clusters: Three Candidate Z 9-10 Galaxies and Improved Constraints on the Star Formation Rate Density at Z 9.2, ApJ 795 (Nov., 2014) 126, [1211.2230].

[2] H. Liszt and R. Lucas, Galactic OH absorption and emission toward a sample of compact extragalactic mm-wave continuum sources., A\&A 314 (Oct., 1996) 917-926.

[3] C. Heiles and T. H. Troland, The Millennium Arecibo 21 Centimeter Absorption-Line Survey. II. Properties of the Warm and Cold Neutral Media, ApJ 586 (Apr., 2003) 1067-1093, [arXiv:astro-ph/0207105].

[4] M. G. Wolfire, C. F. McKee, D. Hollenbach and A. G. G. M. Tielens, Neutral Atomic Phases of the Interstellar Medium in the Galaxy, ApJ 587 (Apr., 2003) 278-311, [arXiv:astro-ph/0207098].

[5] C. Ledoux, P. Noterdaeme, P. Petitjean and R. Srianand, Neutral atomic-carbon quasar absorption-line systems at $z \geq 1.5$. Sample selection, $H$ i content, reddening, and $2175 \AA$ extinction feature, A\&A $\mathbf{5 8 0}$ (Aug., 2015) A8, [1504.07254].

[6] N. Kanekar, C. L. Carilli, G. I. Langston, G. Rocha, F. Combes, R. Subrahmanyan et al., Constraints on Changes in Fundamental Constants from a Cosmologically Distant $\mathrm{OH}$ Absorber or Emitter, Physical Review Letters 95 (Dec., 2005) 261301, [astro-ph/0510760].

[7] H. Rahmani, R. Srianand, N. Gupta, P. Petitjean, P. Noterdaeme and D. A. Vásquez, Constraining the variation of fundamental constants at $z \sim 1.3$ using 21 -cm absorbers, MNRAS 425 (Sept., 2012) 556-576, [1206.2653].

[8] J. Darling, Toward a Direct Measurement of the Cosmic Acceleration, ApJL 761 (Dec., 2012) L26, [1211 . 4585].

[9] H. R. Kloeckner, D. Obreschkow, C. Martins, A. Raccanelli, D. Champion, A. L. Roy et al., Real time cosmology - A direct measure of the expansion rate of the Universe with the SKA, Advancing Astrophysics with the Square Kilometre Array (AASKA14) (Apr., 2015) 27, [1501.03822].

[10] N. Gupta, R. Srianand, P. Petitjean, J. Bergeron, P. Noterdaeme and S. Muzahid, Search for cold gas in strong $\mathrm{Mg}$ II absorbers at $0.5 \lesssim z \lesssim 1.5$ : nature and evolution of $21-\mathrm{cm}$ absorbers, A\&A 544 (Aug., 2012) A21, [1205.4029]. 
[11] N. Kanekar, J. X. Prochaska, A. Smette, S. L. Ellison, E. V. Ryan-Weber, E. Momjian et al., The spin temperature of high-redshift damped Lyman $\alpha$ systems, MNRAS 438 (Mar., 2014) 2131-2166, [1312.3640].

[12] F. Combes, Molecular absorptions in high-z objects, Ap\&SS 313 (Jan., 2008) 321-326, [astro-ph/0701894].

[13] J. R. Allison, M. A. Zwaan, S. W. Duchesne and S. J. Curran, Using 21 cm absorption surveys to measure the average H I spin temperature in distant galaxies, MNRAS 462 (Oct., 2016) 1341-1350, [1607.04828].

[14] R. Srianand, N. Gupta, H. Rahmani, E. Momjian, P. Petitjean and P. Noterdaeme, Parsec-scale structures and diffuse bands in a translucent interstellar medium at $z \sim 0.079$, MNRAS 428 (Jan., 2013) 2198-2206, [1210 . 3036].

[15] J. S. Farnes, S. P. O'Sullivan, M. E. Corrigan and B. M. Gaensler, Faraday Rotation from Magnesium II Absorbers toward Polarized Background Radio Sources, ApJ 795 (Nov., 2014) 63, [1406.2526].

[16] P. Noterdaeme, P. Petitjean, C. Ledoux, S. López, R. Srianand and S. D. Vergani, $A$ translucent interstellar cloud at $z=2.69 . \mathrm{CO}, \mathrm{H}_{2}$, and $\mathrm{HD}$ in the line-of-sight to SDSS J123714.60+064759.5, A\&A $\mathbf{5 2 3}$ (Nov., 2010) A80+, [1 008.0637$].$

[17] A. C. Fabian, Observational Evidence of Active Galactic Nuclei Feedback, ARA\&A 50 (Sept., 2012) 455-489, [1204 .4114].

[18] E. Glikman, D. J. Helfand, R. L. White, R. H. Becker, M. D. Gregg and M. Lacy, The FIRST-2MASS Red Quasar Survey, ApJ 667 (Oct., 2007) 673-703, [0 706.3222 ].

[19] W. A. Baan, A. D. Haschick, D. Buckley and J. T. Schmelz, Hydroxyl absorption in NGC 520, NGC 2623, and NGC 6240, ApJ 293 (June, 1985) 394-399.

[20] N. Gupta, C. J. Salter, D. J. Saikia, T. Ghosh and S. Jeyakumar, Probing radio source environments via HI and OH absorption, MNRAS 373 (Dec., 2006) 972-992, [arXiv:astro-ph/0605423].

[21] K. Geréb, F. M. Maccagni, R. Morganti and T. A. Oosterloo, The HI absorption "Zoo", A\&A 575 (Mar., 2015) A44, [1411.0361].

[22] F. M. Maccagni, R. Morganti, T. A. Oosterloo, K. Geréb and N. Maddox, Kinematics and physical conditions of $H \mathrm{I}$ in nearby radio sources. The last survey of the old Westerbork Synthesis Radio Telescope, A\&A 604 (Aug., 2017) A43, [1705. 00492$].$

[23] R. Morganti, C. N. Tadhunter and T. A. Oosterloo, Fast neutral outflows in powerful radio galaxies: a major source of feedback in massive galaxies, A\&A 444 (Dec., 2005) L9-L13, [astro-ph/0510263]. 
[24] E. V. Ryan-Weber, A. Begum, T. Oosterloo, S. Pal, M. J. Irwin, V. Belokurov et al., The Local Group dwarf Leo T: HI on the brink of star formation, MNRAS 384 (Feb., 2008) 535-540, [0711.2979].

[25] J. Darling and R. Giovanelli, The OH Megamaser Luminosity Function, ApJ 572 (June, 2002) 810-822, [astro-ph/0204195].

[26] E. de Blok, F. Fraternali, G. Heald, B. Adams, A. Bosma and B. Koribalski, The SKA view of the Neutral Interstellar Medium in Galaxies, Advancing Astrophysics with the Square Kilometre Array (AASKA14) (Apr., 2015) 129.

[27] D. Obreschkow, M. Meyer, A. Popping, C. Power, P. Quinn and L. Staveley-Smith, The SKA as a Doorway to Angular Momentum, Advancing Astrophysics with the Square Kilometre Array (AASKA14) (Apr., 2015) 138, [1501.01048].

[28] A. Popping, M. Meyer, L. Staveley-Smith, D. Obreschkow, G. Jozsa and D. J. Pisano, Observations of the Intergalactic Medium and the Cosmic Web in the SKA era, Advancing Astrophysics with the Square Kilometre Array (AASKA14) (Apr., 2015) 132, [1501.01077].

[29] S. W. Henderson, R. Allison, J. Austermann, T. Baildon, N. Battaglia, J. A. Beall et al., Advanced ACTPol Cryogenic Detector Arrays and Readout, Journal of Low Temperature Physics 184 (Aug., 2016) 772-779, [1510.02809].

[30] Z. S. Yuan, J. L. Han and Z. L. Wen, The Scaling Relations and the Fundamental Plane for Radio Halos and Relics of Galaxy Clusters, ApJ 813 (Nov., 2015) 77, [1510 . 04980].

[31] R. Cassano, G. Brunetti, R. P. Norris, H. J. A. Röttgering, M. Johnston-Hollitt and M. Trasatti, Radio halos in future surveys in the radio continuum, A\&A 548 (Dec., 2012) A100, [1210.1020].

[32] L. Rudnick and F. N. Owen, The Distribution of Polarized Radio Sources $>15 \mu J y$ in GOODS-N, ApJ 785 (Apr., 2014) 45, [1402.3637].

[33] J.-P. Uzan, The fundamental constants and their variation: observational and theoretical status, Reviews of Modern Physics 75 (Apr., 2003) 403-455, [hep-ph / 0205340 ].

[34] J. B. Whitmore and M. T. Murphy, Impact of instrumental systematic errors on fine-structure constant measurements with quasar spectra, MNRAS 447 (Feb., 2015) 446-462, [1 409 . 4467].

[35] P. Lah, J. N. Chengalur, F. H. Briggs, M. Colless, R. de Propris, M. B. Pracy et al., The HI content of star-forming galaxies at $z=0.24$, MNRAS 376 (Apr., 2007) 1357-1366, [arXiv:astro-ph/0701668].

[36] N. Kanekar, J. X. Prochaska, S. L. Ellison and J. N. Chengalur, A search for $H I 21 \mathrm{~cm}$ absorption in strong MgII absorbers in the redshift desert, MNRAS 396 (June, 2009) 385-401, [0903.4487]. 
[37] S. J. Curran, P. Tzanavaris, J. K. Darling, M. T. Whiting, J. K. Webb, C. Bignell et al., New searches for HI $21 \mathrm{~cm}$ in damped Lyman $\alpha$ absorption systems, MNRAS 402 (Feb., 2010) 35-45, [0910.3742].

[38] R. Srianand, N. Gupta, P. Petitjean, P. Noterdaeme, C. Ledoux, C. J. Salter et al., Search for cold gas in $z>2$ damped Ly $\alpha$ systems: 21 -cm and $\mathrm{H}_{2}$ absorption, MNRAS 421 (Mar., 2012) 651-665, [1112.1438].

[39] N. Gupta, R. Srianand, D. V. Bowen, D. G. York and Y. Wadadekar, GMRT mini-survey to search for 21-cm absorption in quasar-galaxy pairs at $z \sim 0.1$, MNRAS 408 (Oct., 2010) 849-864, [1 007.0288$]$.

[40] S. Borthakur, T. M. Tripp, M. S. Yun, E. Momjian, J. D. Meiring, D. V. Bowen et al., Using $21 \mathrm{~cm}$ Absorption in Small Impact Parameter Galaxy-Quasar Pairs to Probe Low-redshift Damped and Sub-damped Lyo Systems, ApJ 713 (Apr., 2010) 131-145, [0 912 . 2575].

[41] S. N. Reeves, E. M. Sadler, J. R. Allison, B. S. Koribalski, S. J. Curran, M. B. Pracy et al., H I emission and absorption in nearby, gas-rich galaxies - II. Sample completion and detection of intervening absorption in NGC 5156, MNRAS 457 (Apr., 2016) 2613-2641, [1601.03753].

[42] R. Dutta, N. Gupta, R. Srianand and J. M. O'Meara, Mapping kiloparsec-scale structures in the extended H I disc of the galaxy UGC 000439 by H I 21-cm absorption, MNRAS 456 (Mar., 2016) 4209-4218, [1601.00971].

[43] R. Braun, Cosmological Evolution of Atomic Gas and Implications for $21 \mathrm{~cm} \mathrm{H} \mathrm{I}$ Absorption, ApJ 749 (Apr., 2012) 87, [1202.1840].

[44] N. Gupta, R. Srianand, P. Petitjean, P. Noterdaeme and D. J. Saikia, A complete sample of 21-cm absorbers at z 1.3: Giant Metrewave Radio Telescope survey using MgII systems, MNRAS 398 (Sept., 2009) 201-220, [0 904 . 2878].

[45] L. Staveley-Smith and T. Oosterloo, HI Science with the Square Kilometre Array, Advancing Astrophysics with the Square Kilometre Array (AASKA14) (Apr., 2015) 167, [1506.04473].

[46] R. Morganti, E. M. Sadler and S. Curran, Cool Outflows and HI absorbers with SKA, Advancing Astrophysics with the Square Kilometre Array (AASKA14) (Apr., 2015) 134, [1501.01091].

\section{A. Author Affiliations}

${ }^{1}$ Inter-University Centre for Astronomy and Astrophysics, India; ${ }^{2}$ ASTRON, the Netherlands Institute for Radio Astronomy, The Netherlands; ${ }^{3}$ Rutgers, the State University of New Jersey, USA; ${ }^{4}$ University of 
Manchester, UK; ${ }^{5}$ National Radio Astronomy Observatory, USA; ${ }^{6}$ Laboratoire d'Astrophysique de Marseille, France; ${ }^{7}$ Physics and Astronomy Department, University of Western Cape, South Africa; ${ }^{8}$ Observatoire de Paris, France; ${ }^{9}$ Centre for High Performance Computing, South Africa; ${ }^{10}$ Dark Cosmology Center, Niels Bohr Institute, Denmark; ${ }^{11}$ CSIRO Astronomy and Space Science, Australia; ${ }^{12}$ Astrophysics and Cosmology Research Unit, University of KwaZulu Natal, South Africa; ${ }^{13}$ Astrophysics, University of Oxford, UK; ${ }^{14}$ Square Kilometre Array South Africa, South Africa; ${ }^{15}$ National Centre for Radio Astrophysics, India; ${ }^{16}$ Argelander-Institut für Astronomie (AIfA), Universität Bonn, Germany; ${ }^{17}$ Max-Planck-Institut für Radioastronomie, Germany; ${ }^{18}$ Institut d'Astrophysique de Paris, France; ${ }^{19}$ University of South Carolina, Department of Physics and Astronomy, USA; ${ }^{20}$ European Southern Observatory, Chile; ${ }^{21}$ Astronomy Department, California Institute of Technology, USA; ${ }^{22}$ South African Astronomical Observatory, South Africa; ${ }^{23}$ INAF-Osservatorio Astronomico di Cagliari, Italy; ${ }^{24}$ Department of Physics, Royal Military College of Canada, Canada; ${ }^{25}$ Kapteyn Astronomical Institute, University of Groningen, The Netherlands; ${ }^{26}$ University of Utah, USA; ${ }^{27}$ International Centre for Radio Astronomy Research, The University of Western Australia, Australia. 